\title{
Ingestión y excreción de fluoruros en niños de Hermosillo, Sonora, México
}

\author{
María Isabel G rijalva-Haro, Q .B., M. en C., ${ }^{(1)}$ María Elena Barba-Leyva, Q .B., ${ }^{(1)}$ \\ Arturo Laborín-Alvarez, Q.B. ${ }^{(2)}$
}

\section{Grijalva-Haro, MI, Barba-Leyva ME, Laborín-Alvarez A. Ingestión y excreción de fluoruros en niños de Hermosillo, Sonora, México. Salud Publica Mex 2001;43:127-134.}

EI texto completo en inglés de este artículo está disponible en: http://www.insp.mx/salud/index.html

\section{Resumen}

Objetivo. Estimar la ingestión de fluoruros $\left(\mathrm{F}^{-}\right)$y su relación con la excreción urinaria en un grupo de niños escolares de 8 y 9 años de edad. Material y métodos. En un estudio transversal se evaluaron 31 niños del sexo masculino, residentes de la ciudad de Hermosillo, Sonora, México, y vecinos de tres colonias abastecidas con agua de diferentes concentraciones de fluoruros, de febrero a noviembre de 1997. La ingestión de F- se estimó mediante el registro y medición del consumo de agua y de la colección de un duplicado de los alimentos consumidos durante un periodo de 24 horas. La excreción de F- se midió en una muestra de orina de 24 horas. La concentración de éstos se determinó por el método de potenciómetro de ion selectivo. Resultados. La concentración de fluoruros en el agua de consumo fue de $2.77,0.78$ y $0.54 \mathrm{mg} / \mathrm{l}$, para las colonias Bugambilias, Cuauhtémoc y Balderrama, respectivamente, encontrándose diferencias significativas $(p<0.01)$. La ingestión media de fluoruros para la colonia con más alto nivel fue de 5.41 $\mathrm{mg} / \mathrm{día}$, mientras que para las restantes la ingestión media fue de 2.31 y de $1.51 \mathrm{mg} / \mathrm{día}$. De los niños estudiados, 32\% muestran valores de ingestión por arriba de la recomendación y de éstos $60 \%$ provienen de la colonia con el nivel de F- más elevado. El consumo de agua representó $63 \%$ de la ingestión total de $\mathrm{F}$, y la ingestión media de agua fue de $1.8 \mathrm{l} / \mathrm{dí}$. La excreción media de F- fue de $1.66 \mathrm{mg} / 24 \mathrm{~h}$ ( $\mathrm{ran}$ go: 0.18 - $8.18 \mathrm{mg} / 24 \mathrm{~h}$ ). El análisis de regresión múltiple

\author{
Grijalva-Haro, MI, Barba-Leyva ME, \\ Laborín-Alvarez A. \\ Fluoride intake and excretion among \\ children in Hermosillo, Sonora, Mexico. \\ Salud Publica Mex 2001;43:127-134. \\ The English version of this paper \\ is available at: http://www.insp.mx/salud/index.html
}

(1) Dirección de Nutrición. Centro de Investigación en Alimentación y Desarrollo, A.C., Hermosillo, Sonora, México.

(2) Instituto Mexicano del Seguro Social, Ciudad Juárez, Chihuahua, México.

Fecha de recibido: 17 de junio de 1999 - Fecha de aprobado: 31 de julio de 2000

Solicitud de sobretiros: María Isabel Grijalva Haro. Dirección de N utrición. Centro de Investigación en Alimentación y Desarrollo, A.C. C arretera a la Victoria Km 0.6, 83200 A partado Postal 1735, Hermosillo, Sonora, México.

Correo electrónico: grijalva@ cascabel.ciad.mx 
por pasos mostró una asociación entre los fluoruros proveniente de agua y la excreción urinaria de fluoruros $\left(R^{2}=\right.$ $0.56 ; p=0.001$ ). Conclusiones. La ingestión de fluoruros en la colonia con alto nivel de éstos, representa más de dos veces la recomendación diaria para este grupo de edad; asimismo, se encontró que la concentración de $F^{-}$en el agua fue el principal aportador de este elemento en la dieta. El texto completo en inglés de este artículo está disponible en: http://www.insp.mx/salud/index.html

Palabras clave: concentración de fluoruros; ingestión de fluoruros; excreción de fluoruros; niño; México
Fluoride intake in the neighborhood with the highest fluoride level was twice the daily recommended value for 8-9 year-old children. D rinking water was the main source of fluoride intake. The English version of this paper is available at: http://www.insp.mx/salud/index.html

Key words: fluo ride level; fluo ride intake; fluoride excretion; child; Mexico
E 1 flúor $\left(\mathrm{F}^{-}\right)$es considerado como un elemento traza potencialmente tóxico con algunas funciones bioquímicas indispensables, ya que se requiere para la formación de tejido óseo y para el mantenimiento de la integridad ósea. ${ }^{1,2}$ Existen reportes controversiales sobre el beneficio de los fluoruros en la prevención de caries dental, pues se ha considerado un factor importante en la disminución de su prevalencia. ${ }^{1-3}$ Sin embargo, la excesiva ingestión por periodos prolongados causa toxicidad la cual se manifiesta con la aparición de fluorosis dental, fluorosis esquelética y fracturas de cadera. Recientemente, estudios toxicológicos con animales han asociado daños en los niveles neurológico y reproductivo. ${ }^{4,5}$

Por otro lado, existen informes provenientes de comunidades que cuentan con agua fluorada o sin fluorar, que señalan que ha habido un incremento en la ocurrencia de fluorosis moderada en niños. ${ }^{2-7} \mathrm{La}$ explicación a esto es que se ha aumentado el consumo de $\mathrm{F}^{-}$debido a la utilización de productos tales como dentífricos, enjuagues y suplementos, así como un incremento de $\mathrm{F}^{-}$en la cadena alimenticia, ${ }^{4,8}$ y particularmente en México la sal fluorada. La ingestión total depende de: la concentración de $\mathrm{F}^{-}$en el agua de consumo, uso de agua hervida, alimentos, edad del individuo y condiciones ambientales (temperaturas elevadas), las cuales influyen en el consumo de líquidos y en la dieta. ${ }^{9-11}$

El cálculo de la ingestión diaria de $\mathrm{F}^{-}$no resulta ser una tarea fácil, ya que no existe información suficiente sobre el contenido de éstos en alimentos, además de la falta de estudios sobre $\mathrm{F}^{-}$en la dieta que consume la población y de estudios de balance. ${ }^{12,13}$

En Sonora el contenido de $\mathrm{F}^{-}$en el agua de consumo de fuentes naturales difiere grandemente en un rango de 0.2 a $7.82 \mathrm{mg} / 1^{14,15} \mathrm{y}$, dependiendo de la región, se tienen temperaturas máximas anuales que van de 36 a $41^{\circ} \mathrm{C}$ en los meses de abril a septiembre.

De acuerdo con la recomendación dada por Gallagan y Vermillion, ${ }^{16}$ para climas con temperaturas entre 32 y $37.5^{\circ} \mathrm{C}$, la concentración óptima en el agua debe ser de $0.6 \mathrm{mg} / 1 .^{5}$ Un estudio realizado en San Luis Potosí, Mexico, ${ }^{9}$ mostró que la prevalencia y severidad de fluorosis dental encontrada en los niños fue debido a un incremento en la concentración de $\mathrm{F}^{-}$ en el agua. Los factores de riesgo fueron: temperatura ambiente, agua hervida y el uso de agua hervida para la preparación y cocinado de los alimentos. El factor de riesgo más importante para determinar si la fluorosis dental o esquelética puede ocurrir, así como su severidad, es la determinación de la ingestión total de fluoruros de todas las fuentes. ${ }^{4,17}$ Se ha informado que la ingestión de grandes cantidades de fluoruros (5-20 mg/día) pueden producir severas deformidades en el esqueleto ${ }^{4,5}$ y se ha observado que en

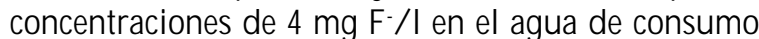
existe un mayor riesgo de fracturas de cadera. ${ }^{1}$ El efecto tóxico de los fluoruros depende no únicamente del tiempo de exposición, sino que también se encuentra asociado a otros factores como son el estado nutricional, función renal e interacciones con otros elementos, particularmente calcio.

En este estudio el objetivo fue estimar la ingestión total y excreción urinaria de fluoruros en niños de 8 y 9 años, del sexo masculino, en tres colonias con diferentes concentraciones de fluoruros en el agua de consumo.

\section{Material y métodos}

En un estudio previo realizado en el estado de Sonora se identificó al municipio de Hermosillo por su amplio rango en concentraciones de $\mathrm{F}^{-}$en el agua de consumo. ${ }^{14,15}$ Por lo anterior, el presente trabajo se llevó a cabo en tres colonias de la ciudad de Hermosillo, Sonora, México, que presentaron diferentes concentraciones de fluoruros en el agua de consumo: Balderrama, localizada en el centro de la ciudad, con $0.54 \pm 0.29 \mathrm{mg}$ $\mathrm{F}^{-} / \mathrm{l}$; Cuauhtémoc, en el sur $\left(0.78 \pm 0.0 \mathrm{mg} \mathrm{F}^{-} / 1\right)$ y Bugambilias, en el norte, la cual presentó niveles de 
$2.77 \pm 0.49 \mathrm{mg} \mathrm{F}^{-} / 1$. Este estudio fue de corte transversal y se llevó a cabo de febrero a noviembre de 1997.

Se acudió a tres escuelas públicas localizadas cada una en los sectores seleccionados. Se realizó un muestreo intencional no probabilístico, en el que participaron 31 niños en edades de entre 8 y 9 años, del sexo masculino. Los padres fueron visitados en sus domicilios y firmaron voluntariamente las formas de consentimiento de participación en el estudio. El requisito de ingreso fue residencia mínima de cinco años en el sector, corroborándose con la matrícula de la escuela a donde los niños asistieron a clases.

Para llevar a cabo esta fase del estudio, se visitó el domicilio y se instruyó a la madre y al niño; a éste se le proporcionó un recipiente de plástico (capacidad 1.51 ) con diseños infantiles adecuados para motivarlo, para que tomara agua exclusivamente del recipiente durante un periodo de $24 \mathrm{~h}$. El envase se llenó y se pesó con el agua que el niño ingiere normalmente; este procedimiento se repitió cuantas veces fue necesario, registrándose el dato en el cuaderno proporcionado para el diario de alimentos. La ingestión promedio diaria de agua fue el peso total del agua ingerida por el niño. Para el análisis de $\mathrm{F}^{-}$se tomó una muestra del agua que el niño consumía y del agua utilizada en el cocinado de los alimentos.

Se proporcionó a la madre una balanza digital (capacidad de $1 \mathrm{Kg}$. Ohaus, modelo Lume O Meter, New Jersey, Estados Unidos de América -EUA-), recipientes de plástico ( capacidad 1 l) y un diario de alimentos para el registro de los mismos. La colección del duplicado ${ }^{18}$ del total de alimentos consumidos durante el día, se realizó con la colaboración de la madre o el niño, los cuales pesaron exactamente igual la cantidad consumida (en su forma preparada, considerando la sal y el agua utilizada) y colectaron el duplicado de los alimentos y bebidas ingeridas por el niño durante el periodo de $24 \mathrm{~h}$. Este proceso se repitió cada vez que el niño consumió algo durante el día, ya fuera alimentos, golosinas o bebidas. Cada porción de alimento consumido (duplicado) se colocó en un mismo recipiente. Posteriormente, el total de alimentos colectados fueron homogeneizados en una licuadora (Waring, Blendor, modelo 34BC22, EUA), se tomó una alícuota de $100 \mathrm{~g}$ y se congeló a $-20{ }^{\circ} \mathrm{C}$ hasta su análisis.

Los alimentos consumidos (g) por el niño durante un periodo de $24 \mathrm{~h}$, se pesaron y registraron en el diario. ${ }^{19}$ Se anotó, además, la descripción, los ingredientes y las cantidades utilizadas en la preparación de los alimentos. Con base en el registro de alimentos se calculó la frecuencia de consumo de alimentos, con- siderando así el número de veces que aparece el alimento, número de registros y la cantidad promedio de consumo de los mismos.

Una muestra de orina de 24 horas, se colectó en recipientes de plástico. Todo el material utilizado, previamente fue lavado, enjuagado y remojado en ácido nítrico $(20 \% \mathrm{p} / \mathrm{v})$. Todas las muestras se recogieron en cada casa "a primera hora" de la mañana.

$\mathrm{El}$ análisis de $\mathrm{F}^{-}$en alimentos se realizó de acuerdo a la técnica de difusión de fluoruros con hexametildisiloxano (HMDS, Sigma, EUA), descrita por Singer y Ophaug. ${ }^{20}$ Los análisis de F- en agua y orina se realizaron siguiendo las técnicas de Frant y $\operatorname{Ross}^{21}$ y Tusl ${ }^{22}$ (para orina se utilizó $9 \mathrm{ml}$ de muestra y $1 \mathrm{ml}$ de acetato de sodio a 15\%, Merck, México). Después se agregó la solución Tisab en relación 1:1 tanto en agua como en orina. Antes de la lectura en el potenciómetro se ajustó $1 \mathrm{ph}$ (4.5-5.5).

Para la determinación de F- se utilizó un potenciómetro de ion selectivo (Corning, modelo 350, Corning Incorporated, Nueva York 14831, EUA), un electrodo selectivo de F- (Corning, modelo 476135, Corning Incorporated, Nueva York 14831, EUA ) y un electrodo de referencia de doble unión (Corning, modelo 476370, Corning Incorporated, Nueva York 14831, EUA).

La técnica para determinar la concentración de Fen alimentos se validó para su precisión y exactitud con el método de adición de estándares. Se utilizó como muestra atún enlatado obteniéndose una concentración de $0.441 \pm 0.012 \mathrm{mg} / \mathrm{kg}$ con $98.4 \%$ de recuperación. Para el análisis de agua y orina se utilizó un estándar de referencia certificado (Enviromental Resource Associates, Arvada, Colorado, EUA), donde se obtuvo una concentración promedio de $4.26 \pm 0.27 \mathrm{mg} / 1$ contra el valor señalado para el agua certificada que es de $4.28 \mathrm{mg} / \mathrm{l}$ (rango de 3.64 - 4.92mg/l)

Se calculó la media y la desviación estándar de la ingestión de fluoruros provenientes del agua, los alimentos y la excreción urinaria. Se realizó un análisis de varianza, se probó la homogeneidad de acuerdo con la prueba de Bartlett's y se llevó a cabo la prueba no paramétrica de Kruskal-Wallis para observar diferencias entre las colonias. Asimismo, se realizó un análisis de regresión lineal simple, donde se incluyó la excreción urinaria de $\mathrm{F}^{-}$como variable dependiente, y la concentración $\mathrm{F}^{-}$en agua, ingestión proveniente del consumo de agua, concentración de $\mathrm{F}^{-}$en alimentos, la ingestión por alimentos y la ingestión total de F- como variables independientes; además se realizó un análisis de correlación. Las variables que presentaron una relación significativa fueron puestas en un modelo de regresión múltiple por pasos, con el fin de obtener 
la variable que presentara el mayor efecto en la excreción urinaria de F-. Para la realización de las pruebas se utilizó el paquete estadístico Statgraphics, Versión 4.0, 1989 .

\section{Resultados}

En el cuadro I, se presenta la comparación de los valores obtenidos sobre la concentración de F- en el agua; ingestión de agua (ml/día); la ingestión de F- proveniente de agua; del total de alimentos consumidos y que fueron preparados y cocinados en su forma tradicional; la ingestión total (agua más alimentos incluyendo sal y bebidas); y excreción urinaria de $\mathrm{F}^{-}$en niños de 8 a 9 años de tres colonias con diferente concentración de fluoruros en Hermosillo, Sonora. Para estimar la concentración óptima de $\mathrm{F}^{-}$en el agua es necesario considerar la temperatura media máxima anual y para los climas cálidos se recomienda una concentración de $0.6 \mathrm{mg} / 1 .{ }^{15}$ Con base en lo anterior, la colonia Bugambilias, situada en el norte de la ciudad, presentó la mayor concentración de fluoruros en el agua: $361 \%$ arriba de la concentración óptima recomendada; mientras que para la colonia Cuauhtémoc (sur) se encontró 30\% arriba, y una concentración menor (10\% debajo del óptimo) se observó para la colonia Balderrama (centro). Sin embargo, si consideramos la Norma Oficial Mexicana (máximo $1.5 \mathrm{mg} / \mathrm{l}$ ), la colonia Bugambilias excede con $84.7 \%$, mientras que la Cuauhtémoc y la Balderrama se encuentra por debajo en $47.7 \%$ y $64.0 \%$, respectivamente. Se encontraron diferencias significativas entre las colonias $(p<0.01)$.

El consumo promedio de agua fue de $1875 \mathrm{ml} /$ día (rango de 1 a 3 1/día), y no se encontró diferencia estadísticamente significativa en la ingestión de agua por colonia $(p>0.05)$.
La ingestión de $\mathrm{F}^{-}$, considerando únicamente el consumo de agua, fue mayor para la colonia Bugambilias y menor para la colonia Balderrama $(p<0.05)$.

Con respecto a la ingestión de $\mathrm{F}^{-}$proveniente de alimentos se observó una tendencia a un consumo más elevado en la colonia Bugambilias, aunque éste no fue diferente estadísticamente con respecto a las otras dos colonias $(p>0.01)$, quizás por el tamaño de muestra que participó en este estudio. Sin embargo, cuando se consideró la ingestión total de $\mathrm{F}^{-}$(consumo de agua más alimentos), la cantidad consumida en la colonia Bugambilias fue mayor de las otras colonias $(p<0.05)$.

La cantidad de $\mathrm{F}^{-}$ingerida se reflejó en la excreción urinaria, donde los valores más elevados se presentaron en la colonia Bugambilias $(p<0.01)$.

En la figura 1 se presentan los porcentajes de ingestión total de fluoruros de acuerdo con la cantidad considerada como segura y adecuada, ${ }^{23}$ que es de 1.5 a $2.5 \mathrm{mg} /$ día para niños de 7 a 11 años. Se observa que $60 \%$ de los niños que ingirieron $\mathrm{F}^{-}$por arriba de la recomendación provenían de la colonia Bugambilias.

Con base en el registro de alimentos, los más frecuentemente consumidos y sus cantidades fueron: leche $(290 \mathrm{ml})$, frijol $(117 \mathrm{~g})$, huevo $(70 \mathrm{~g})$, cereal $(38 \mathrm{~g})$, pan $(43 \mathrm{~g})$, carne $(67 \mathrm{~g})$, bebidas preparadas en casa (polvos con saborizantes, $378 \mathrm{ml})$, tortilla de maíz $(45 \mathrm{~g})$, papas fritas (71g), tortilla de harina (58g); así también un menor consumo de refrescos de lata $(235 \mathrm{ml})$; el consumo de frutas y verduras fue muy bajo. Los valores de $\mathrm{F}^{-}$obtenidos para los refrescos analizados en el laboratorio fueron los siguientes: los refrescos de sabores en lata estuvieron en el rango de 0.46 a $0.60 \mathrm{y}$ para los refrescos de cola variaron de 0.51 a $1.10 \mathrm{mg} \mathrm{F}^{-} / 1$.

El análisis de regresión simple efectuado (figuras 2, 3, y 4) mostró que existe una fuerte asociación entre la concentración de $\mathrm{F}^{-}$en el agua de consumo y la ex-

Cuadro I

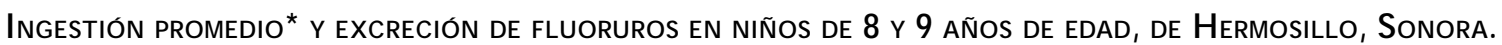
Centro de Investigación y Desarrollo, A.C., Hermosillo, Sonora, México, 1997

\begin{tabular}{|c|c|c|c|c|}
\hline & $\begin{array}{c}\text { Bugambilias } \\
(n=10)\end{array}$ & $\begin{array}{c}\text { Cuauhtémoc } \\
(n=10)\end{array}$ & $\begin{array}{c}\text { Balderrama } \\
(n=/ I)\end{array}$ & $p$ \\
\hline Concentración de $\mathrm{F}^{-}$en el agua de consumo (mg/l) & $2.77^{\ddagger} \pm 0.49$ & $0.784^{\ddagger} \pm 0.00$ & $0.54^{\ddagger} \pm 0.29$ & $<0.01$ \\
\hline Ingestión de agua (ml/d) & $1875 \pm 766$ & $2063 \pm 1812$ & $1568 \pm 542$ & NS \\
\hline Ingestión de F- por agua (mg/d) & $3.69 \ddagger \pm 3.26$ & $1.61^{\ddagger} \pm 1.41$ & $0.77^{\ddagger} \pm 0.49$ & $<0.05$ \\
\hline Ingestión de F- por alimentos (mg/d) & $1.72 \pm 2.25$ & $0.706 \pm 0.76$ & $0.73 \pm 0.79$ & NS \\
\hline Ingestión total de $\mathrm{F}^{-}$(mg/d) & $5.41^{\ddagger} \pm 4.83$ & $2.32^{\ddagger} \pm 1.64$ & $1.51^{\ddagger} \pm 1.11$ & $<0.05$ \\
\hline Excreción de F- (mg/d ) & $3.10^{\ddagger} \pm 2.02$ & $1.04^{\ddagger} \pm 0.46$ & $0.93^{\ddagger} \pm 0.53$ & $<0.001$ \\
\hline
\end{tabular}




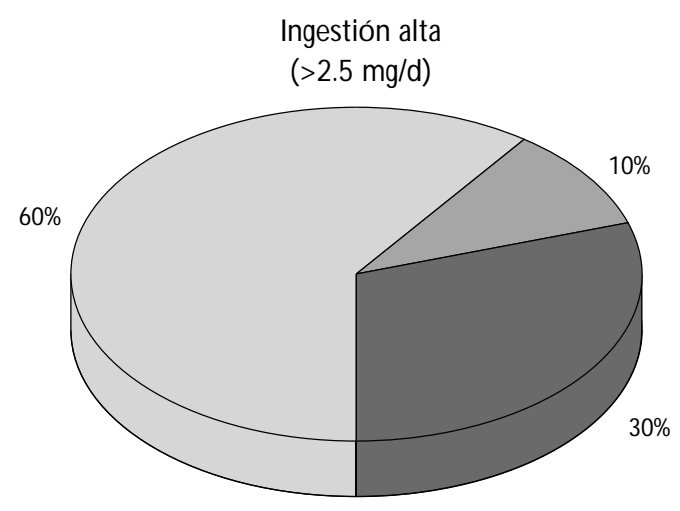

Ingestión adecuada

$(1.5-2.5 \mathrm{mg} / \mathrm{d})$
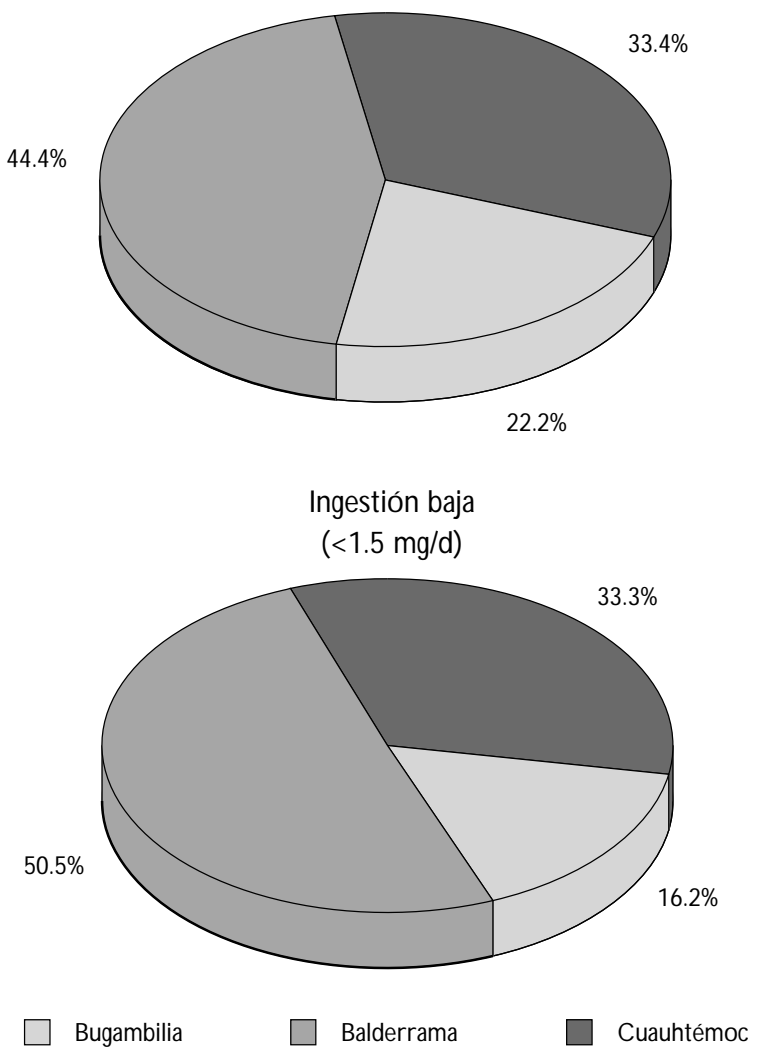

Figura 1. Distribución de la ingestión de fluoruROS, CON bASE EN LA RECOMENDACIÓN DE LA NATIONAL Academy of Science de Estados Unidos de AmérlCa. ${ }^{23}$ Centro de Investigación y Desarrollo, A.C., Hermosillo, Sonora, México, 1997

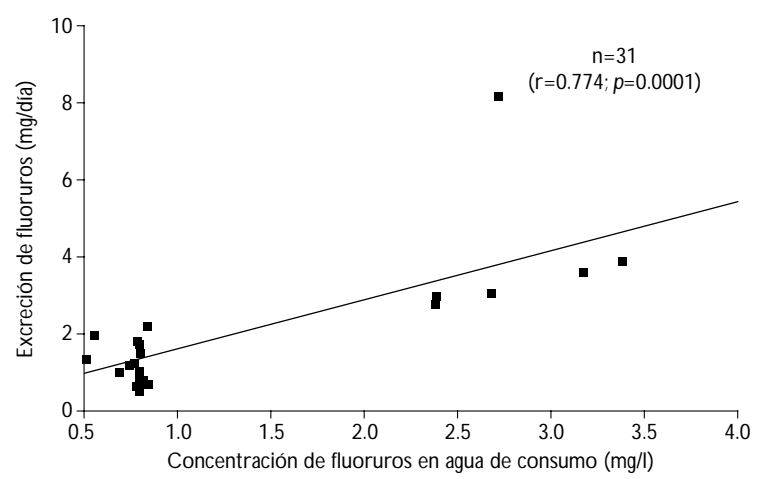

Figura 2. Relación de la concentración de fluoRUROS EN EL AGUA Y SU EXCRECIÓN, EN NIÑOS DE 8 Y 9 años de la ciudad de Hermosillo, Sonora, México. Centro de Investigación y Desarrollo, A.C ., 1997

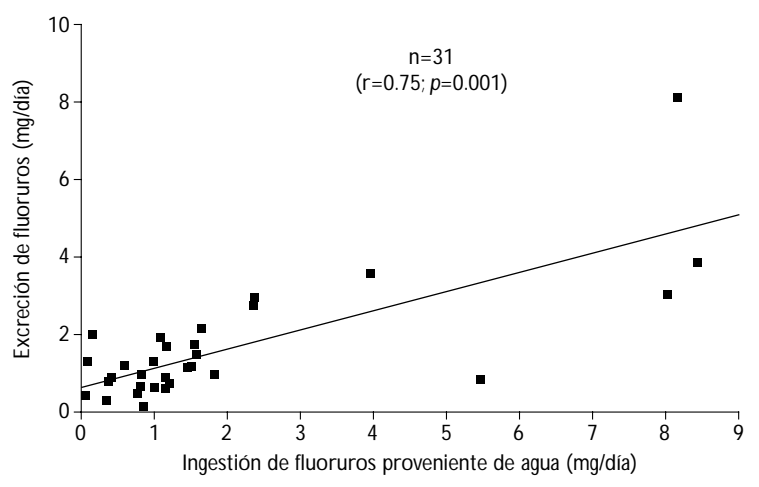

Figura 3. Relación de la ingesta de fluoruros ProVENIENTES DE AGUA Y SU EXCRECIÓN, EN NIÑOS DE 8 Y 9 años de la ciudad de Hermosillo, Sonora, México. Centro de Investigación y Desarrollo, A.C., 1997

creción urinaria de $\mathrm{F}^{-}(\mathrm{r}=0.774 ; p<0.001)$, para la ingestión de $\mathrm{F}^{-}$proveniente de agua $(\mathrm{r}=0.750 ; p<0.001) \mathrm{y}$ la ingestión total de $\mathrm{F}^{-} \mathrm{y}$ la excreción urinaria $(\mathrm{r}=0.67$; $p<0.001$ ). Todas las variables estudiadas se incluyeron en un modelo de regresión múltiple por pasos y resultó que la ingestión de $\mathrm{F}^{-}$por consumo de agua fue 


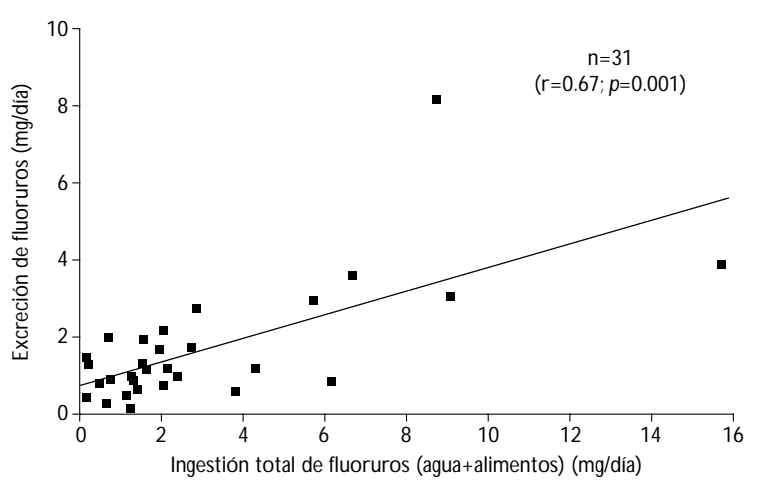

Figura 4. Relación entre la ingestión total de fluoRUROS Y SU EXCRECIÓN, EN NIÑOS DE 8 Y 9 AÑOS DE LA ciudad de Hermosillo, Sonora, México. Centro de InVESTIGACIÓn y DeSARRolLo, A.C., 1997

la variable que mejor explicó la variación en la excreción urinaria $\left(R^{2}=0.56 ; p=0.001\right)$.

\section{Discusión}

En relación con a la concentración de $\mathrm{F}^{-}$en el agua de consumo la colonia Bugambilias, localizada al norte de la ciudad, presentó la mayor concentración de $\mathrm{F}^{-}$, casi dos veces el límite máximo permisible de 1.50 $\mathrm{mg} / \mathrm{l}$ con respecto a la Norma Oficial Mexicana. ${ }^{24} \mathrm{De}$ acuerdo con la Organización Mundial de la Salud, ${ }^{10}$ se recomienda que en climas templados la concentración de $\mathrm{F}^{-}$en el agua de consumo se restrinja de 0.5 a $0.7 \mathrm{mg} / \mathrm{l}$, que es el límite máximo permitido para aguas purificadas y envasadas (Norma Oficial Mexicana). ${ }^{25}$ En este estudio se observó que el agua de las colonias Cuauhtémoc y Balderrama se encuentran en este rango, por lo que se pudiera considerar que estos niveles serían lo mas cercano a lo adecuado para la ciudad de Hermosillo, Sonora.

Con respecto a un estudio anterior sobre concentraciones de fluoruros en el agua de consumo en el estado de Sonora, ${ }^{14}$ se observó que las concentraciones de $\mathrm{F}^{-}$en las colonias estudiadas variaron de acuerdo con lo encontrado en este estudio. Así, por ejemplo, en la colonia Bugambilias los niveles de $\mathrm{F}^{-}$eran de $5.85 \mathrm{mg} / 1$ y ahora son de $2.77 \pm 0.49 \mathrm{mg} / \mathrm{l}$. Lo anterior se debe a que el organismo regulador del agua potable (Coapaes) ha realizado interconexiones en las redes de los suministros de agua con el fin de disminuir la concentración de fluoruros en las colonias del norte de Hermosillo. En el periodo que se realizó el presente trabajo, se estuvieron monitoreando los niveles de $\mathrm{F}^{-} \mathrm{y}$ éstos se observaron en un rango de 2.37 a $3.70 \mathrm{mg} / \mathrm{l}$.

En lo referente al consumo de agua, en un estudio $^{26}$ realizado en el estado Sonora, en los municipios de Puerto Peñasco, Alamos, Nacozari y Hermosillo, en niños de 6 a 12 años de los dos sexos, se encontró que el consumo promedio de agua fue de $1.37 \pm 0.82$ l/día, valor ligeramente menor al encontrado en este estudio $(1.87 \pm 1.411 /$ día). Lo anterior es importante cuando se tiene que considerar la recomendación $\mathrm{n}^{5,16}$ dada para encontrar la concentración óptima de fluoruros en el agua, ya que se debe considerar además de la temperatura promedio máxima anual, el volumen de agua ingerida, por un incremento en la ingestión de líquidos en los climas cálidos con el consiguiente aumento en la ingestión de fluoruros. ${ }^{27}$

Como se muestra en el cuadro I, el mayor aporte de $\mathrm{F}^{-}$fue proveniente de agua de consumo, ya que el aporte por alimentos fue menor en las tres colonias. También se observó que en la colonia Bugambilias fue donde se tuvo el mayor consumo de $\mathrm{F}^{-}$tanto en el agua como en alimentos, y esto probablemente se debió a la preparación y cocinado de los alimentos con el agua de mayor concentración de $\mathrm{F}^{-}$, y concuerda con lo reportado en estudio realizado en San Luis Potosí, ${ }^{9}$ donde se manifiesta que hervir el agua aumenta la concentración de $\mathrm{F}^{-}$por efecto de evaporación. Por otro lado, el efecto de la concentración de $\mathrm{F}^{-}$en el agua se reflejó en la excreción urinaria, ya que también en la misma colonia Bugambilias se obtuvieron los valores más elevados de excreción.

La recomendación de ingestión total de fluoruros es de 1.5 a $2.5 \mathrm{mg} /$ día. ${ }^{23}$ Esta cantidad es considerada la adecuada para prevenir tanto la caries como la fluorosis dental. De acuerdo con este estudio, se encontró que para la colonia Bugambilias la cantidad estimada de la ingestión de fluoruros fue de $5.41 \mathrm{mg} /$ día; es decir, más de dos veces la recomendación. Lo anterior pudiera explicar el problema de fluorosis dental que actualmente se observa en la mayoría de los niños del sector norte de la ciudad de Hermosillo. Para el caso de las colonias Balderrama y Cuauhtémoc se tiene una ingestión promedio de acuerdo con la recomendación para el grupo de edad de los niños estudiados.

Es importante mencionar que de acuerdo con la recomendación, del total de niños estudiados, $32 \%$ de ellos tuvieron una ingestión promedio elevada de fluoruro, o sea, mayor de $2.5 \mathrm{mg} /$ día (rango de 2.7 $15.8 \mathrm{mg} /$ día); $29 \%$ consumieron cantidades adecuadas entre 1.5-2.4 mg/día, y 39\% ingirieron cantidades por debajo de la recomendada para este grupo de edad. Respecto a estos resultados (figura 1), los niños que 
consumieron mayor cantidad de $\mathrm{F}^{-}, 60 \%$ de ellos provenía de la colonia Bugambilias; $30 \%$, de la Cuauhtémoc, y sólo 10\%, de la Balderrama, con lo cual se refleja el efecto de la concentración de $\mathrm{F}^{-}$en el agua de consumo. Cabe aclarar que durante el estudio cuatro niños de la Bugambilias reportaron que habían dejado de consumir agua de la llave, para que sus dientes ya no se mancharan y también algunos niños consumen agua procesada o purificada, y dependiendo de la marca, la concentración de $\mathrm{F}^{-}$en este tipo de agua varió de 0.058 a $1.09 \mathrm{mg} / \mathrm{l}$.

En general, el agua aportó en promedio $63 \%$ del $\mathrm{F}^{-}$consumido por los niños de este estudio y el resto por alimentos. Cabe aclarar que en este estudio se trato de estimar el consumo de $\mathrm{F}^{-}$proveniente de la sal; sin embargo, uno de los principales problemas para realizar esta medición fue el que las madres preparan los alimentos para toda la familia, con lo cual no se pudo estimar su aporte como tal, aunque se detectó que la sal que consumían tenía la etiqueta de sal yodada y fluorada. La OMS recomienda que en lugares donde el contenido natural de $\mathrm{F}^{-}$en agua es mayor de $0.5 \mathrm{mg} / 1$, no debe comercializarse la sal fluorada. De acuerdo con el Diario Oficial de la Federación ${ }^{28}$ la ciudad de Hermosillo aparece dentro de las localidades donde no deberá comercializarse este tipo de sal, sin embargo, este producto se expende en los establecimientos comerciales, lo que en un futuro pudiera agravar el problema de fluorosis dental.

El consumo promedio (g) de los alimentos en este estudio, concuerda con lo reportado en la canasta de consumo de alimentos para el estado de Sonora. ${ }^{29}$ Por otro lado, y con base en la frecuencia de consumo y al tipo de alimentos consumidos éstos son bajos aportadores de fluoruros de acuerdo a lo reportado en la literatura, ${ }^{30}$ en el rango de $0.05 \mathrm{a} 0.31 \mathrm{mg} / \mathrm{l}$. Se ha documentado un elevado consumo de refrescos en el país; con respecto a este estudio, $33 \%$ de los niños ingirieron este tipo de bebidas y el consumo promedio fue de $235 \mathrm{ml}$; por lo anterior, se estimó el aporte de fluoruros por este alimento, resultando ser bajo, ya que fue de $0.145 \mathrm{mg} / \mathrm{l}$.

En cuanto a la excreción urinaria de $\mathrm{F}^{-}$, se observó el marcado efecto de proporcionalidad que tuvo la concentración de éstos en el agua, esto concuerda con el estudio realizado por Valenzuela, ${ }^{26}$ donde observó esta misma asociación; lo cual sucedió igual para la ingestión total de $\mathrm{F}^{-}$(agua + alimentos) y la excreción, donde se encontró que a mayor consumo de $\mathrm{F}^{-}$, mayor excreción. Estos resultados son similares a los de otros estudios reportados,,$^{30}$ donde al medir la concentración de fluoruros en agua y orina se observó que el nivel de
$\mathrm{F}^{-}$en el agua fue directamente proporcional a la concentración de $\mathrm{F}^{-}$en orina. Los valores de excreción de $\mathrm{F}^{-}$(3.10 mg/día) en la colonia Bugambilias coinciden con los reportados por Maheshwari y colaboradores, ${ }^{13}$ quienes al suplementar con $5 \mathrm{mg}$ de fluoruro de sodio encontraron una excreción dentro del rango de 3.10 a $4.12 \mathrm{mg} /$ día.

Con los resultados de este estudio, se podría inferir que para la ciudad de Hermosillo, Sonora, el agua de consumo es el principal vector o la fuente principal para el aporte de $\mathrm{F}^{-}$en la dieta de los sujetos estudiados y no los alimentos que componen la dieta.

Por otro lado, cabe mencionar que se tuvieron algunos $(n=6)$ casos de niños que tuvieron una ingestión baja de $\mathrm{F}^{-}$y una elevada excreción. Esto concuerda con estudios realizados por Largent, ${ }^{31}$ donde demostró la movilización de los fluoruros, ya que al consumir suplementos de $\mathrm{F}^{-}$por dos años consecutivos y después, al dejar de tomar este suplemento, encontró un balance negativo. Lo anterior pudiera sugerir que en los niños que han estado expuestos por periodos prolongados al consumo de $\mathrm{F}^{-}$, y que han dejado de ingerir cantidades elevadas, su remoción a través de la orina se ve aumentada. Los casos encontrados en este estudio fueron en niños que estuvieron ingiriendo aguas purificadas que tuvieron concentraciones en el rango de 0.05 a $0.50 \mathrm{mg} / \mathrm{l}$.

\section{Conclusiones}

En Hermosillo, Sonora, las concentraciones de fluoruros en el agua de consumo son variables. La colonia Bugambilias presentó una concentración de dos veces el límite máximo permitido por la Norma Oficial Mexicana. De acuerdo al clima y temperatura de esta ciudad se considera que la concentración óptima debe ser de 0.5-0.7 mg/l. De acuerdo a esta consideración, las colonias Cuauhtémoc y Balderrama cumplen con este rango de concentración. El consumo promedio de agua fue de 1.81 y este fue el principal vehículo aportador de $\mathrm{F}^{-}$, ya que contribuyó con $63 \%$ de la ingestión total de este nutrimento.

La cantidad de fluoruros proveniente de los alimentos fue variable y en ellos se reflejó la concentración de $\mathrm{F}^{-}$en el agua con los cuales fueron cocinados, como fue el caso de la colonia Bugambilias, donde se encontró mayor aporte por alimentos.

En las colonias Cuauhtémoc y Balderrama la ingestión total de fluoruros estuvo dentro de la recomendación para el grupo de edad estudiado y no ocurre lo mismo para la colonia Bugambilias donde se consumió el doble de la recomendación. 
La ingestión total de fluoruros en $32 \%$ de los niños excedió la recomendación y de éstos $60 \%$ provienen de la colonia Bugambilias.

La excreción de fluoruros fue afectada por la concentración de fluoruros en el agua, por la ingestión de $\mathrm{F}^{-}$proveniente de agua y por la ingestión total de $\mathrm{F}^{-}$.

Debido a los resultados obtenidos en este estudio se recomienda a las autoridades de salud y a los organismos reguladores y abastecedores de agua, buscar fuentes alternativas o mezclas adecuadas con el fin de que se disminuya el contenido de fluoruros en el agua que abastece al sector norte de Hermosillo, ya que de no hacerlo se agravará el problema de fluorosis dental que ya se observa en los niños de esa área. Asimismo, se recomienda que no se permita la distribución de sal fluorada. Por otro lado, es importante realizar un estudio sobre la prevalencia de caries y fluorosis dental y otros posibles desórdenes o enfermedades.

De este modo, se hace necesario continuar la investigación determinando y comparando todas las fuentes de ingestión de fluoruros incluyendo los productos dentales.

\section{Referencias}

1. World Health $\mathrm{O}$ rganization. Fluoride. En: W HO. Trace elements in human nutrition and health. Ginebra: W HO . 1996:187-194.

2. World Health Organization. Fluorides and oral health. W HO Expert Committee on 0 ral health Status and Fluoride Use. G inebra: W HO, 1994; Technical Report Series 846:6-15.

3. Szpunar SM, Burt BA. D ental caries, fluorosis, and fluoride exposure of Michigan school children. J Dent Res 1988;67:802-806.

4. D epartment of Health and Human Services. Review of fluoride: Benefits and risks. Report of the Ad Hoc Subcommittee on Fluoride of the Committee to Coordinate Environmental Health and Related Programs. W ashington, D.C.: Public Health Service, 1991, Public Health Service 1991. 5. N ational Research C ouncil. Health effects of ingested fluoride. Subcommittee on Health Effects of Ingested Fluoride. W ashington. D.C ., N ational A cademy Press 1993

6. Ekstrand J. Fluoride intake in early infancy. J N ut 1989;19:1856-1860.

7. Adair SM, Leverett DH, Shields CP. Fluoride content of school lunches from an optimal fluoridated and a fluoride deficient community. J Food Composition Analysis 1991;4:216-226.

8. Leverett DH. Prevalence of dental fluorosis in fluoridated and nonfluoridate communities. A preliminary investigation. J Public Health Dent 1986;46:184-187.

9. Grimaldo M, Borja-Aburto VH, Ramírez AL, Ponce M, Rosas M, DíazBarriga F. Endemic fluorosis in San Luis Potosí, México. Environ Res 1995; 68:25-30.

10. Murray J. A ppropriate use of fluoride for human health. Ginebra: W orld Health $O$ rganization, 1986;3-25.

11. Heargraves JA. W ater fluoridated and fluoride supplementation: Considerations for future. J D ent Res 1990;69(special issue): 765-770.
12. Ekstrand J, Hardell L, Spack C J. Fluoride balances studies on infants in a 1ppm water-fluoride-area. Caries Res 1984;18:87-92.

13. Maheshwari VR, MCD onald JT, Schneider VS, Brunetti AJ, Leybin L, N ewbrun $\mathrm{E}$ et al. Fluoride balance studies in ambulatory healthy men with and without fluoride supplements. Am J C lin N utr 1981;34:2679-2684.

14. G rijalva MI, Valenzuela A I, Silveira MI, Benítez MA . La concentración de fluoruros en el agua y su efecto en la salud dental en el estado de Sonora. Estudios Sociales 1998;III(15):111-134.

15. Díaz-Barriga F, N avarro-Q uezada A, Grijalva MI, G rimaldo M, LoyolaRodriguez JP, Ortiz MD. Endemic fluorosis in Mexico. Fluoride 1997; 30(4):233-239.

16. Gallagan DJ, Vermillion J R. Determining optimum fluoride concentration. Public Health Rep 1957;72(6):491-493.

17. A gency for Toxic Substances and D isease Registry. Toxicological profile for fluoride, hydrogen fluoride, and fluoride. Department of Health and Human Services, Public Health 1993.

18.G rijalva MI, Valencia ME, W yatt CJ. Sodium, potassium, and calcium intake in adults consuming normal diets in Northern Mexico determined by analytical and calculated methods. J Food Composition Analysis 1992:5:127-133

19. Bingham SA. The dietary assessment of individuals: Methods, accuracy, new techniques and

recommendations. Dietary Survey Methodology 1987;10:705-741.

20. Singer $L, O$ phaug RH. D etermination of fluoride in foods. J A gric Food Chem 1986;34(3):510-513.

21. Frant MS, Ross JW. U se of a total ionic strength, adjustment buffer for electrode determination of fluoride water supplies. Anal Chem 1968; 40(7):1169-1171.

22. Tusl J. Fluoride ion activity electrode a suitable means for exact determination of urinary fluoride. Anal Chem 1972;44(9):1693-1694.

23. $N$ ational Academy of Science. Recommended dietary allowances. Décima edición. W ashington, D.C. N ational A cademy Press, 1989.

24. N orma $O$ ficial Mexicana: N O M-127-SSAI-1994, Salud A mbiental. A gua para uso y consumo humano. Límites permisibles de calidad y tratamientos a que debe someterse el agua para su potabilización. México, D.F.: Diario 0 ficial de la Federación CVIII 13, 18 ene. 1996:6.

25. N orma 0 ficial Mexicana: N O M-041-SSA I-1993, Bienes y Servicios. A gua purificada envasada. Especificaciones sanitarias. México, D.F.: Diario 0 ficial de la Federación CDXCVIII 18, 24 mar. 1995:52.

26. Valenzuela AI. N iveles de flúor en el agua de consumo humano y su relación con la salud dental del estado de Sonora (tesis de Maestría). Hermosillo, Sonora: Centro de Investigación en Alimentación y Desarrollo, A.C., 1992.

27. Fejerskov 0, Manji F, Baelum V. The nature and mechanisms of dental fluorosis in man. J Dent Res 1990; 69 (Special issue): 692-700.

28. Diario 0 ficial de la Federación. Listado mediante el cual se dan a conocer las áreas por entidad federativa donde "no" debe comercializarse sal yodada y fluorada, por tener el agua de consumo una concentración de flúor natural mayor de $0.7 \mathrm{mg} /$. México, D.F. jueves 11 de abril de 1996:68-70 (primera sección).

29. Valencia ME, Hoyos LC, Ballesteros MN, 0 rtega MI, Palacios MR, Atondo JL. La dieta en Sonora: canasta de consumo de alimentos. Estudios Sociales 1998;8(15):12-39.

30. Singer $\mathrm{L}, \mathrm{O}$ phaug $\mathrm{RH}, \mathrm{H}$ arland $\mathrm{BF}$. Fluoride intake of young male adults in the United States. Am. J Clin N utr 1980;33:28-332.

31. W hitford GM. The physiological and toxicological characteristics of fluoride. J Dent Res

1990; 69 (Special issue): 539-549.

32. Largent EJ. Rates of elimination of fluoride stored in tissues of man. Arch Indust Hyg 1952; 6:37-42. 